\title{
Upper Limb Soft Tissue Necrotic Lesion
}

National Cancer Institute

\section{Source}

National Cancer Institute. Upper Limb Soft Tissue Necrotic Lesion. NCI Thesaurus. Code C78621.

A necrotic process affecting the soft tissues of the upper extremity. 